\title{
Antibacterial Activity of Coumarine Derivatives Synthesized from 4-Chloro-chromen-2-one. The Comparison with Standard Drug
}

\author{
AZIZBEHRAMI \\ Faculty of Food Technology, University of Mitrovica, Kosovo. \\ *Corresponding author E-mail: a.behrami_chem@live.com \\ http://dx.doi.org/10.13005/ojc/300433 \\ (Received: August 05, 2014; Accepted: September 26, 2014)

\begin{abstract}
This work reports the synthesis of some new derivatives from 4-Chloro-chromen-2-one and describe the results of antibacterial activity of purified compounds. Compounds 4-Butylaminochromen-2-one (1a) , 4-Butylamino-2-oxo-2H-chromene-3-sulfonyl chloride (2a) , 4-Butylamino2-oxo-2H-chromene-3-sulfonic acid (2-hydroxy-phenyl)-amide (3a), 4-Butylamino-5-ethyl-2-oxo7-(N'-phenyl-hydrazino)-2H-chromene-3-sulfonic acid (2-hydroxy-phenyl)-amide (4a), have been synthesized and characterized using melting points, IR spectra, ${ }^{1} \mathrm{H}-\mathrm{NMR}$ and ${ }^{13} \mathrm{C}$-NMR spectra. The antibacterial activity of synthesized compounds and streptomycin at concentractions of $1 \mathrm{mg} /$ $\mathrm{ml}, 3 \mathrm{mg} / \mathrm{ml}$ and $5 \mathrm{mg} / \mathrm{ml}$, have been evaluated against three strains of bacterial culture; Staphylococcus aureus, E.coli and Klebsiella. The compounds show bacteriostatic and bactericidal activity.
\end{abstract}

Key words: 4-Chloro-chromen-2-one, Coumarine derivatives, Antibacterial activity, Staphylococcus aureus, E.coli, Klebsiella, Streptomycin.

\section{INTRODUCTION}

Starting from 4-Chloro-chromen-2-one (a); derivatives $(1 a, 2 a, 3 a, 4 a)$ are synthesized

Coumarin derivatives are large group of heterocyclic with oxygen as heteroatom (Govori et al 1996 ; Govori et al 2002 ; Stanovnik et al 1993).Coumarin is a chemical sompound (specifically , a benzo- $\alpha$-pyrone) found in many plants (Lee et al 2002) notably in high concentration in the tonka bean ( Dipteryx odorata), vanilla grass
(Anthoxanthum odoratum), woodruff (Galium odoratum), mullein (Verbascum spp), and sweet grass (Hierochloe odorata).Coumarine and their derivatives have shown varius biological activities. Their fame has come mainly from their antithrombic, antiinflammatory, vasodilatory, and antiviral activities. Other several coumarin derivatives have antimicrobial properties (Sanghyun ; et al 1996 ; Mohareb et al 2007; Nofal et al 2000), have urged us to synthesize some new coumarin derivatives and to investigate their antibacterial activity against 
staphylococcus aureus, E.coli and Klebsiella.The antibacterial activity of synthesized compounds is compared with antibacterial activity of streptomycin.

\section{MATERIAL AND METHODS}

\section{Experimental Chemistry}

Compounds 4-Butylamino-chromen-2one (1a) , 4-Butylamino-2-oxo-2H-chromene-3sulfonyl chloride (2a), 4-Butylamino-2-oxo-2Hchromene-3-sulfonic acid (2-hydroxy-phenyl)amide (3a), 4-Butylamino-5-ethyl-2-oxo-7-(N'phenyl-hydrazino)-2H-chromene-3-sulfonic acid (2hydroxy-phenyl)-amide (4a), are synthesized.

The identification of $2 \mathrm{H}$-chromen-2-one derivatives $(1 \mathrm{a}, 2 \mathrm{a}, 3 \mathrm{a}, 4 \mathrm{a})$, is made by using melting point, infrared, ${ }^{1} \mathrm{H}$ NMR,${ }^{13} \mathrm{C}$ NMR spectra and elemental analysis. Melting point was determinated on a Electrothermal apparatus (Fisher Scientific 2555) in a open capillary tube and are uncorrected.Infrared spectra were recorded in $\mathrm{cm}^{-1}$ for $\mathrm{KBr}$ pellts on a FT-IR Shimadzu $8400 \mathrm{~S}$ spectrophotometer with resolution $4 \mathrm{~cm}^{-1} .{ }^{1} \mathrm{H}$ NMR spectra were recorded on a Bruker UNITY plus-<smiles>O=c1cc(Cl)c2ccccc2o1</smiles>

4-Chloro-chromen-2-one

a
500 'NMR 1' spectrometer using DMSO- $d_{6}$ as the solvent and TMS as the internal references standard $(\sigma=0,00 \mathrm{ppm})$.Chemical shifts are expressed in ' ppm.Mass spectra were taken on a LKB 9000 mass spectrometer.

Element analysze was performed on a Perikin-Elmer $240 \mathrm{BCHN}$ analyzer.The purity of the compounds (synthesized) was routinely checked by TLC using Merck Kieselgel-60 (F-254) and benzene,toluene,glacial acetic acid (80:10:10)as mobile phase. The spots were exposed in iodine vapour for visualization.

\section{Synthesis of 4-Butylamino-chromen-2-one (1a)}

For this synthesis is used as substrat 4Chloro-chromen-2-one in a $100 \mathrm{ml}$ flask mixed $3 \mathrm{~g}$ of 4-Chloro-chromen-2-one with $8 \mathrm{ml} \mathrm{C}_{2} \mathrm{H}_{5} \mathrm{OH}$, equivalent amount Butylamino. The mixture was refluxed at $250{ }^{\circ} \mathrm{C}$ for ca. $90 \mathrm{~min}$. The obtained crystals brown are filtred and rinsed with ethanol and dried at room temperature.Recrystallization form absolute ethanol gave a red product of $80 \%$ yield, melting point $117^{\circ} \mathrm{C}$.(Sheme 1)

Scheme 1: Synthesis of Compounds 4-Butylamino-chromen-2-one (1a)

Synthesis of 4-Butylamino-2-oxo-2H-chromene3-sulfonyl chloride (2a)

In a $100 \mathrm{ml}$ flask were mixed $2.5 \mathrm{~g}$ of 4 Butylamino-chromen-2-one, with $5 \mathrm{ml} \mathrm{CH}_{3} \mathrm{CN}, 1 \mathrm{ml}$ $\mathrm{ClSO}_{3} \mathrm{H}, 0.3 \mathrm{ml} \mathrm{Et}_{3} \mathrm{~N}$.

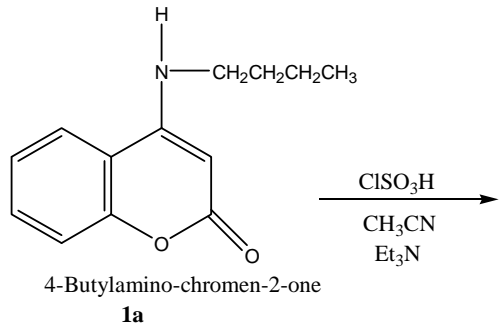

The mixture was refluxed at $80^{\circ} \mathrm{C}$ for ca. $1.5 \mathrm{~h}$. The obtained brown crystals are filtred and dried at room temperature . Recrystallization form $\mathrm{C}_{2} \mathrm{H}_{5} \mathrm{OH}$ gave brown crystals product of $70 \%$ yield,meltingpoint, $287^{\circ} \mathrm{C}$. (Scheme 2 ) .

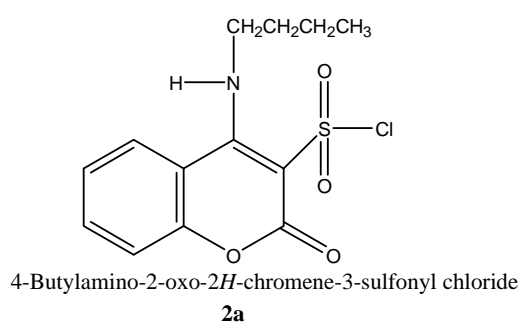

Scheme 2 - Synthesis of 4-Butylamino-2-oxo-2H-chromene-3-sulfonyl chloride (2a) 
Synthesis of 4-Butylamino-2-oxo-2H-chromene3-sulfonic acid (2-hydroxy-phenyl) -amide (3a) In a $100 \mathrm{ml}$ flask were mixed $1.5 \mathrm{~g} \mathrm{4-}$ Butylamino - 2 - oxo - 2H- chromene - 3- sulfonyl chloride with $4 \mathrm{ml}$ Dioxane and $1 \mathrm{~g}$ aminophenol, $0.2 \mathrm{ml} \mathrm{HCl}, 0,2 \mathrm{ml} \mathrm{Et}_{3} \mathrm{~N}$ as katalyzer. The mixture was refkuxed at $92{ }^{\circ} \mathrm{C}$ in water bath for ca. $2 \mathrm{~h}$. The flask was placed in an ice bath for $1 \mathrm{~h}$ until yellow crystalline precipitate was formed.

After filtration the product was recrystallized from ethanol.The recrystallizacion from ethanol gave a yellow product at $70 \%$ yield, melting.point; $180^{\circ} \mathrm{C}$. (Scheme 3 ).

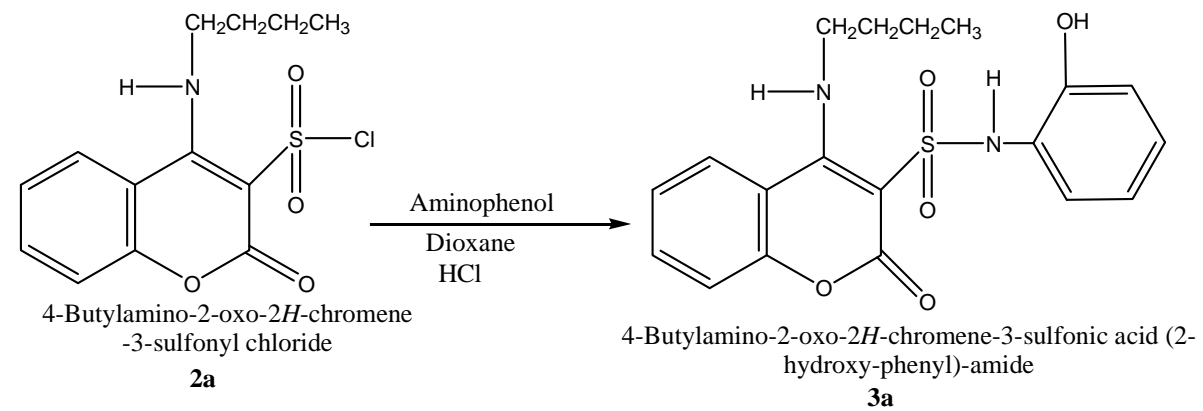

Scheme 3: Synthesis of 4-Butylamino-2-oxo-2H-chromene-3-sulfonic acid (2-hydroxy-phenyl) -amide (3a)<smiles>CCCNc1c(S(=O)(=O)Nc2ccccc2O)c(=O)oc2ccccc12</smiles>

4-Butylamino-2-oxo-2H-chromene-3-sulfonic acid (2-hydroxy-phenyl)-amide
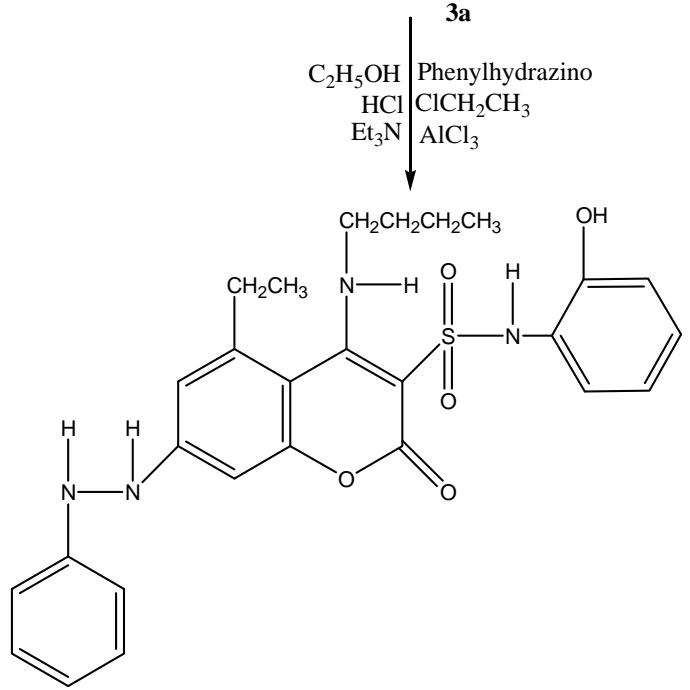

4-Butylamino-5-ethyl-2-oxo-7-( $N$ '-phenyl-hydrazino)-2H-chromene-3-sulfonic acid (2-hydroxyphenyl)-amide

4a

Scheme 4: Synthesis of 4-Butylamino-5-ethyl-2-oxo-7-(N'-phenyl-hydrazine) -2H-chromene-3-sulfonic acid (2-hydroxy-phenyl)-amide (4a) 
Table 1: Antibacterial activity- Staphylococcus aureus and the comparison with Streptomycine

\begin{tabular}{lccc}
\hline \multirow{2}{*}{ Compound } & \multicolumn{3}{c}{ Inhibition zone (mm) } \\
\cline { 2 - 4 } & $2 \mathbf{m g} / \mathbf{m l}$ & 3mg $/ \mathbf{m l}$ & $\mathbf{5 m g / m l}$ \\
\hline $1 \mathrm{a}$ & 10 & 13 & 15 \\
$2 \mathrm{a}$ & 18 & 20 & 24 \\
$3 \mathrm{a}$ & 19 & 21 & 25 \\
$4 \mathrm{a}$ & 11 & 13 & 18 \\
Streptomycine & 20 & 20 & $2010 \boldsymbol{\mu g}$ \\
\hline
\end{tabular}

Table 3: Antibacterial activity Klebsiella and the comparison with Streptomycine

\begin{tabular}{lccc}
\hline Compound & \multicolumn{3}{c}{ Inhibition zone (mm) } \\
\cline { 2 - 4 } & $2 \mathrm{mg} / \mathbf{m l}$ & 3mg $/ \mathbf{m l}$ & $\mathbf{5 m g} / \mathbf{m l}$ \\
\hline $1 \mathrm{a}$ & 12 & 19 & 23 \\
$2 \mathrm{a}$ & 13 & 18 & 25 \\
$3 \mathrm{a}$ & 13 & 19 & 24 \\
$4 \mathrm{a}$ & 10 & 17 & 21 \\
Streptomycine & 23 & 23 & $2310 \mu \mathrm{g}$ \\
\hline
\end{tabular}

Table 2: Antibacterial activity E.coli and the comparison with Streptomycine

\begin{tabular}{lccc}
\hline Compound & \multicolumn{3}{c}{ Inhibition zone (mm) } \\
\cline { 2 - 4 } & $\mathbf{2 m g / m l}$ & 3mg /ml & $\mathbf{5 m g / m l}$ \\
\hline $1 \mathrm{a}$ & 5 & 9 & 14 \\
$2 \mathrm{a}$ & 10 & 15 & 21 \\
$3 \mathrm{a}$ & 12 & 17 & 23 \\
$4 \mathrm{a}$ & 11 & 15 & 20 \\
Streptomycine & 23 & 23 & $2310 \mu \mathrm{g}$ \\
\hline
\end{tabular}

Synthesis of 4 - Butylamino - 5 - ethyl -2 - oxo 7 - ( N' - phenyl - hydrazine )- 2H-chromene-3sulfonic acid (2-hydroxy-phenyl)-amide (4a)

In a $100 \mathrm{ml}$ flask were mixed $1 \mathrm{~g}$ of 4 Butylamino-2-oxo-2H-chromene-3-sulfonic acid (2hydroxy-phenyl) - amide, $0.8 \mathrm{~g}$ phenylhidrazine with $4 \mathrm{ml} \mathrm{C}_{2} \mathrm{H}_{5} \mathrm{OH}, 0.5 \mathrm{ml} \mathrm{ClCH}_{2} \mathrm{CH}_{3} \quad 0.2 \mathrm{ml} \mathrm{Et}_{3} \mathrm{~N}$ and 0.2 $\mathrm{ml} \mathrm{HCl}$. The mixture was refluxed at $95^{\circ} \mathrm{C}$ in water bath for ca. $2 \mathrm{~h}$. The obtained red crystals are filtred and rinsed with $\mathrm{CH}_{3} \mathrm{CN}$ and dried at room temperature.Recrystallization from ethanol gave a

Table 4

\begin{tabular}{|c|c|c|c|}
\hline Compound & $\operatorname{IR}\left(\mathrm{cm}^{-1}\right)$ & ${ }^{1} \mathrm{H}$ NMR ppm & ${ }^{13}$ C NMR ppm \\
\hline $1 \mathrm{a}$ & $\begin{array}{l}3370(\mathrm{NH}), 3010(\mathrm{C}-\mathrm{H}) \text { ar, } \\
2962(\mathrm{C}-\mathrm{H}) \text { alifatic } \\
1720(\mathrm{C}=\mathrm{O}), 1570(\mathrm{C}=\mathrm{C}) \mathrm{ar}, \\
1385(\mathrm{C}-\mathrm{O}), 750(\mathrm{C}-\mathrm{H}) \mathrm{ar}\end{array}$ & 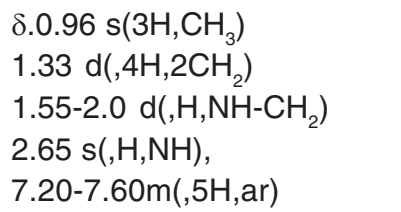 & 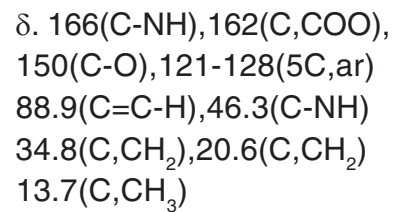 \\
\hline $2 a$ & $\begin{array}{l}3370(\mathrm{~N}-\mathrm{H}), 3008(\mathrm{C}-\mathrm{H}) \text { ar } \\
2960(\mathrm{CH}) \text { alifatic, } \\
1740(\mathrm{C}=\mathrm{O}), 1600(\mathrm{C}=\mathrm{C}) \\
1380\left(\mathrm{SO}_{2} \mathrm{Cl}\right), 1285(\mathrm{C}-\mathrm{O}) \\
720(\mathrm{C}-\mathrm{H}) \mathrm{ar}\end{array}$ & $\begin{array}{l}\delta .0 .96 \mathrm{~s}\left(, 3 \mathrm{H}, \mathrm{CH}_{3}\right) \\
1.33-1.55, \mathrm{~d}\left(4 \mathrm{H}_{2}, \mathrm{CH}_{2}\right) \\
2.65 \mathrm{~s}\left(\mathrm{H}, \mathrm{NHCH}_{2}\right) \\
3.0 \mathrm{~s}(\mathrm{H}, \mathrm{NH}) \mathrm{ar} \\
7.20-7.63 \mathrm{~m}(4 \mathrm{H}, \mathrm{ar})\end{array}$ & 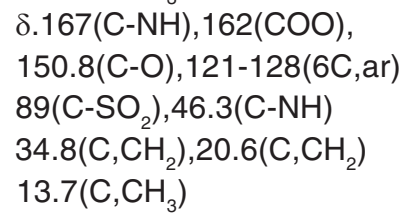 \\
\hline $3 a$ & $\begin{array}{l}3400(\mathrm{OH}), 3300(\mathrm{NH}) \\
3265\left(\mathrm{SO}_{2} \mathrm{NH}\right), 3009 \\
(\mathrm{C}-\mathrm{H}) \mathrm{ar}, 2850(\mathrm{C}-\mathrm{H}) \mathrm{al}, \\
1730(\mathrm{C}=\mathrm{O}), 1528(\mathrm{C}=\mathrm{C}) \mathrm{ar}, \\
1280(\mathrm{~N}-\mathrm{H}), 1275(\mathrm{C}-\mathrm{O}) \\
1250(\mathrm{C}-\mathrm{O}), 740(\mathrm{C}-\mathrm{H}) \mathrm{ar}\end{array}$ & $\begin{array}{l}\delta .0 .96 \mathrm{~s}\left(3 \mathrm{H}, \mathrm{CH}_{3}\right) \\
1.33-1.55 \mathrm{~d}\left(4 \mathrm{H}_{2} \mathrm{CH}_{2}\right) \\
2.65 \mathrm{~s}\left(\mathrm{H}, \mathrm{NHCH} \mathrm{H}_{2}\right) 3.0 \mathrm{~s} \\
(\mathrm{H}, \mathrm{NH}), 4.0 \mathrm{~s}\left(\mathrm{H}_{,} \mathrm{NHSO}_{2}\right) \\
5.0 \mathrm{~s}(\mathrm{H}, \mathrm{OH}) \\
6.29-6.63 \mathrm{~m}(8 \mathrm{H}, \mathrm{ar})\end{array}$ & $\begin{array}{l}\delta .167(\mathrm{C}-\mathrm{NH}), 162(\mathrm{COO}) \\
150(\mathrm{C}-\mathrm{O}), 144(\mathrm{C}-\mathrm{O}), \\
134(\mathrm{C}-\mathrm{NH}), 116-127(9 \mathrm{C}, \mathrm{ar}) \\
46.2(\mathrm{C}-\mathrm{NH}) 20.6\left(\mathrm{C}, \mathrm{CH}_{2}\right) \\
13.7\left(\mathrm{C}, \mathrm{CH}_{3}\right)\end{array}$ \\
\hline $4^{\mathrm{a}}$ & $\begin{array}{l}3387(\mathrm{O}-\mathrm{H}), 3330(\mathrm{~N}-\mathrm{H}) \\
3270\left(\mathrm{SO}_{2} \mathrm{NH}\right), 3010(\mathrm{C}-\mathrm{H}) \mathrm{ar} \\
2900(\mathrm{C}-\mathrm{H}) \mathrm{al}, 1728(\mathrm{C}=\mathrm{O}) \\
1600(\mathrm{C}=\mathrm{C}) \mathrm{ar}, 1280(\mathrm{~N}-\mathrm{H}) \\
1270(\mathrm{C}-\mathrm{O}), 750(\mathrm{C}-\mathrm{H}) \mathrm{ar}\end{array}$ & $\begin{array}{l}\delta .0 .96-1.24 \mathrm{~d}\left(6 \mathrm{H}, 2 \mathrm{CH}_{3}\right) \\
1.33-1.55 \mathrm{~d}\left(4 \mathrm{H}, 2 \mathrm{CH}_{2}\right) 2.0 \mathrm{~s} \\
(\mathrm{H}, \mathrm{NH}), 2.65 \mathrm{~s}(\mathrm{H}, \mathrm{NH}) 2.59 \mathrm{~s} \\
(\mathrm{H}, \mathrm{CH}), 4.0 \mathrm{t}(\mathrm{H}, \mathrm{NH}) 5.0 \mathrm{~s} \\
(\mathrm{H}, \mathrm{OH}) 6.29-7.18 \mathrm{~m}(11 \mathrm{H}, \mathrm{ar})\end{array}$ & $\begin{array}{l}\delta .167(\mathrm{C}-\mathrm{NH}), 162(\mathrm{COO}), 151 \\
(\mathrm{C}-\mathrm{O}), 144(\mathrm{C}-\mathrm{O}), 142(\mathrm{C}-\mathrm{NH}), \\
102-138(17 \mathrm{C}, \mathrm{ar}), 89\left(\mathrm{C}-\mathrm{SO}_{2}\right) \\
46.3(\mathrm{C}-\mathrm{NH}), 22.5\left(\mathrm{C}, \mathrm{CH}_{2}\right) \\
13.7\left(\mathrm{C}, \mathrm{CH}_{3}\right), 10.5\left(\mathrm{C}, \mathrm{CH}_{3}\right)\end{array}$ \\
\hline
\end{tabular}


Table 5: Analytical data

\begin{tabular}{|c|c|c|c|c|c|c|c|c|c|}
\hline \multirow[t]{2}{*}{ Compd } & \multirow[t]{2}{*}{ Yield(\%) } & \multirow[t]{2}{*}{ m.p } & \multirow[t]{2}{*}{ M.F } & \multicolumn{6}{|c|}{ Elemental analysis. Calculated (found) (\%) } \\
\hline & & & & C & $\mathbf{H}$ & $\mathbf{N}$ & 0 & $\mathrm{Cl}$ & $\mathbf{s}$ \\
\hline \multirow[t]{2}{*}{$1 a$} & \multirow[t]{2}{*}{80} & \multirow[t]{2}{*}{$117^{\circ} \mathrm{C}$} & \multirow[t]{2}{*}{$\mathrm{C}_{13} \mathrm{H}_{15} \mathrm{NO}_{2}$} & 71.87 & 6.96 & 6.45 & 14.73 & & \\
\hline & & & & 72.00 & 7.11 & 6.15 & 14.32 & & \\
\hline \multirow[t]{2}{*}{$2 a$} & \multirow[t]{2}{*}{70} & \multirow[t]{2}{*}{$287^{\circ} \mathrm{C}$} & \multirow[t]{2}{*}{$\mathrm{C}_{13} \mathrm{H}_{14} \mathrm{CINO}_{4} \mathrm{~S}$} & 49.45 & 4.47 & 4.44 & 20.27 & 11.23 & 10.15 \\
\hline & & & & 50.00 & 5.00 & 4.11 & 20.00 & 11.00 & 9.80 \\
\hline \multirow[t]{2}{*}{ 3а } & \multirow[t]{2}{*}{70} & \multirow[t]{2}{*}{$180^{\circ} \mathrm{C}$} & \multirow[t]{2}{*}{$\mathrm{C}_{19} \mathrm{H}_{20} \mathrm{~N}_{2} \mathrm{O}_{5} \mathrm{~S}$} & 58.75 & 5.19 & 7.21 & 20.59 & & 8.20 \\
\hline & & & & 60.00 & 4.90 & 7.10 & 19.92 & & 8.00 \\
\hline \multirow[t]{2}{*}{$4 a$} & \multirow[t]{2}{*}{60} & \multirow[t]{2}{*}{$204^{\circ} \mathrm{C}$} & \multirow[t]{2}{*}{$\mathrm{C}_{27} \mathrm{H}_{30} \mathrm{~N}_{4} \mathrm{O}_{5} \mathrm{~S}$} & 62.05 & 5.79 & 10.72 & 15.31 & & 6.14 \\
\hline & & & & 61.50 & 5.20 & 10.0 & 15.00 & & 6.00 \\
\hline
\end{tabular}

red product at $60 \%$ yield, melting point $204{ }^{\circ} \mathrm{C}$. (Scheme 4)

\section{Antibacterial activity}

The purified synthesized compounds (1a,2a,3a,4a) was subjected to test in vitro its antibacterial activity against three bacterial cultures ; Staphylococcus aureus,E.Coli and Klebsiella. Antibacterial activity of compounds was investigated applying the Kirby-Bayer method ${ }^{14}$ or disc method ( $d=5.5 \mathrm{~mm}$ max. capacity $10 \mu \mathrm{g}$ )

\section{CONCLUSION}

From the results the followin conclusion were drawn:The study provides the first evidence that compounds $(1 \mathrm{a}, 2 \mathrm{a}, 3 \mathrm{a}, 4 \mathrm{a})$ obviously inhibit the growth of Staphyllococcus auerus, E.coli and Klebsiella.

The compounds $(\mathbf{1} \mathbf{a}, \mathbf{2} \mathbf{a}, \mathbf{3 a}, \mathbf{4 a})$ compared with the antibacterial activity of Streptomycine in S.aureus, and Klebsiella.

The chemical structures of synthesizen compounds were determined according to extensive NMR experiments and published data.

\section{ACKNOWLEDGEMENTS}

The authors thank Prof.Branko Stanovnik,University of Ljubljana and its laboratory staff for ${ }^{1} \mathrm{H}$ NMR spectrum and elemental analyses.

\section{REFERENCES}

1. S.Govori, V.Kalaj, V.Rapic,L.Kalaj and S.Dakovic,Heterocycel.Commun., 2002; 8,129.

2. B.Stanovnik, H.Susachitzky and E.F.Scriven, Progress in Heterocyclic Chemistry, Pergamon Press, Oxford, 1993; 5,pp.75-146.

3. S.H.Lee, D.-S.Shin, J.-S,Kim, K.-B. Oh and S.S.Kan, Arch. Pharm. Res, 2003; 26.

4. K.B.Vyas, K.S.Nimavat, G.R.Jani and M.V. Hathi, Orbital, 2009; 1, 183.

5. A.Z.Abyshev, V.A.Gimdein, E.V. Semenov, E.M, E.MAgev, A.A. Abdulla - Zade and A.B.Gueseinov,Pharm.Chem.J., 2206; 40.607 .
6. M.D.Aytemir, R.C.Hider, D.D.Erol , M.Ozalp and M.Ekizoglu .Turk.J.chem., 2003; 27 : 445.

7. M.M.El.Saghier,M.B.Naili,B.Kh.Rammash, N.A.Saleh and K.M.Kreddan, Arkivoc, 2007; 83.

8. Z.M.Nofal ,M.El-Zahar and S.Abd EI Karim,Molecules, 2000; 5: 99.

9. Chaluvaraju $\mathrm{KC}$ and Ishwarbhat K.Asian ,J Chem 2008; 20, 4335.

10. Rajan Ra Kali , Jubie S,Grworamma B, and Suresh B,Asian J Chem 2008: 20: 5289.

11. Ali Mohammed Ashraf and Sharayar Mohammed. Boorg Med Chem. Lett 2009; 17: 3314 . 
12. Nofal ZM, El-Zahar M, Abd El-Karim S, Novel coumarin derivatives with expected biological activity, Molecules. 2000; 5: 99113.
13. Vyas KB,Nimavat KS,Jani GR,Hathi MV, Synthesis and microbial activity of coumarin derivatives metal complex: An in vitro evaluation.Orbital, 2009; 1: 183-192. 